\title{
RISK THEORY WITH THE GENERALIZED INVERSE \\ GAUSSIAN LÉVY PROCESS
}

BY

MANuel Morales

\begin{abstract}
Dufresne et al. (1991) introduced a general risk model defined as the limit of compound Poisson processes. Such model is either a compound Poisson process itself or a strictly increasing Lévy process. Their construction is based on a nonnegative non-increasing function $Q$ that governs the jumps of the process. This function, it turns out, is the tail of the Lévy measure of the process. We discuss an illustration of their model using a generalized Inverse Gaussian (GIG) Lévy process. This increasing Lévy process has the gamma and the inverse Gaussian process as particular cases. Although mathematically more complex, the GIG Lévy process keeps some of the nice properties of the simpler gamma process.
\end{abstract}

\section{KEYWORDS}

Risk Theory, Ruin Probabilities, Lévy Process, Gamma Process, Generalized Inverse Gaussian Process.

\section{INTRODUCTION}

Dufresne, Gerber and Shiu (1991) constructed a general aggregate claims process $S$ with independent and stationary increments. They define it in terms of a non-negative and non-increasing function $Q$ defined as

$$
Q(x)=\int_{x}^{\infty} q(s) d s, \quad x>0 .
$$

Moreover, the function $Q$ should be such that

$$
\int_{0}^{\infty} x[-d Q(x)]<\infty
$$

The process $S$ is uniquely defined by its Laplace transform

$$
\mathbb{L}_{t}(z)=\mathbb{E}\left[e^{-z S(t)}\right]=e^{t \Psi(z)}, \quad z>0,
$$


where the exponent $\Psi$ is given by

$$
\Psi(z)=\int_{0}^{\infty}\left[e^{-z x}-1\right][-d Q(x)], \quad z>0 .
$$

This last equation can be recognized as the Laplace exponent of a Lévy process with sample paths of finite variation [see Bertoin (1996) or Sato (1999) for an account on Lévy processes]. The measure $q(d x)=-d Q(x)$ is the Lévy measure of the process $S$. $Q$ denotes then the tail of this Lévy measure.

From the theory of Lévy processes we have that $S$ is either a compound Poisson process (if $Q(0)<\infty$ ) or a process with an infinite number of small jumps (if $Q(0)=\infty$ ). In both cases, the process $S$ can be seen as the limit of a sequence of compound Poisson processes $\left\{S_{\varepsilon}\right\}_{\varepsilon>0}$ described by their Laplace transform

$$
\mathbb{L}_{t}^{(\varepsilon)}(z)=e^{-t \int_{0}^{\infty}\left[e^{-z x}-1\right] d Q_{\varepsilon}(x)}, \quad z>0,
$$

where the measure $Q_{\varepsilon}$ is the restriction to the interval $[\varepsilon, \infty)$ of the tail of Lévy measure $Q$, i.e.

$$
d Q_{\varepsilon}(x)=d Q(x) \rrbracket_{[\varepsilon, \infty)}(x), \quad x>0 .
$$

We can see that

$$
\lim _{\varepsilon \rightarrow 0} d Q_{\varepsilon}(x)=d Q(x), \quad x>0,
$$

which implies that the sequence of compound Poisson processes $\left\{S_{\varepsilon}\right\}_{\varepsilon>0}$ converges weakly in the Skorokhod topology to the process $S$ defined by (2) [see Jacod and Shiryaev (1987)].

Notice that (3) implies that $S$ is a Lévy process with Lévy measure $-d Q(x)$, moreover, condition (1) implies that $S$ is of finite variation.

Dufresne, Gerber and Shiu (1991) explore the process $S$ for

$$
q(d x)=-d Q(x)=x^{-1} e^{-x} d x, \quad x>0,
$$

and

$$
q(d x)=-d Q(x)=x^{-3 / 2} e^{-x} d x, \quad x>0 .
$$

The first choice of $Q$ leads to a gamma process while the second leads to an inverse Gaussian process. These processes are such that their one-dimensional distributions are gamma and inverse Gaussian respectively.

Here we extend this aggregate claims process $S$ to a generalized inverse Gaussian Lévy process (GIG). Such a process is a non-decreasing (subordinator) Lévy process exhibiting the intriguing property of having an infinite number of small jumps. Moreover its increments over periods of length one follow a generalized inverse Gaussian (GIG) distribution. A standard reference on GIG distribution is Jørgensen (1982). The inverse Gaussian process and 
the gamma process of Dufresne, Gerber and Shiu (1991) are particular or limiting cases of the GIG Lévy process, which in turn is another example of the spectrally negative Lévy processes discussed in Yang and Zhang (2001) and of the general risk models discussed in Morales and Schoutens (2003) and Morales (2003).

Section 2 introduces some basic facts about the GIG distribution. In Section 3 we construct the GIG Lévy process and describe some of the properties that make it appealing to ruin modeling. In Section 4, 5 and 6 we discuss ruin theory results for the GIG Lévy process and write down expressions that allows us to compute bounds for the ruin probabilities. Finally, In section 7 we present some numerical illustrations.

\section{Generalized InVerse Gaussian Distribution}

The class of generalized inverse Gaussian distributions is described by three parameters. It has been extensively studied by Jørgensen (1982). The GIG density function has support on the positive axis and is given by

$$
f_{g i g}(x)=\frac{(\gamma / \delta)^{\lambda}}{2 K_{\lambda}(\delta \gamma)} x^{\lambda-1} \exp \left\{-\frac{1}{2}\left(\delta^{2} x^{-1}+\gamma^{2} x\right)\right\}, \quad x>0,
$$

where $K_{\lambda}$ is the modified Bessel function of the third kind with index $\lambda$ given by

$$
K_{\lambda}(x)=\int_{0}^{\infty} u^{\lambda-1} e^{-\frac{1}{2} x\left(u^{-1}+u\right)} d u, \quad x>0 .
$$

The parameter domain of the GIG distribution is

$$
\begin{array}{lll}
\delta>0, & \gamma \geq 0, & \text { if } \lambda<0, \\
\delta>0, & \gamma>0, & \text { if } \lambda=0, \\
\delta \geq 0, & \gamma>0, & \text { if } \lambda>0 .
\end{array}
$$

If $\lambda=-1 / 2$ the density (5) reduces to that of the inverse Gaussian distribution. The gamma distribution is a limiting case of the GIG distribution for $\lambda>0$ and $\gamma>0$ and $\delta \rightarrow 0$. These make the GIG Lévy processes a natural extention to the gamma processes.

The Laplace transform of the GIG is

$$
\mathbb{Z}_{G I G}(z)=\frac{K_{\lambda}\left(\delta \gamma \sqrt{1+\frac{2 z}{\gamma^{2}}}\right)}{K_{\lambda}(\delta \gamma)\left(1+\frac{2 z}{\gamma^{2}}\right)^{-\lambda / 2}},
$$

for $\delta>0$ and $\gamma>0$. Its domain is $z>-\gamma^{2} / 2$ when $\lambda \geq 0$ and $z \geq-\gamma^{2} / 2$ when $\lambda<0$. Figure 1 shows some GIG densities for different values of the parameters. 
$\lambda=-1$

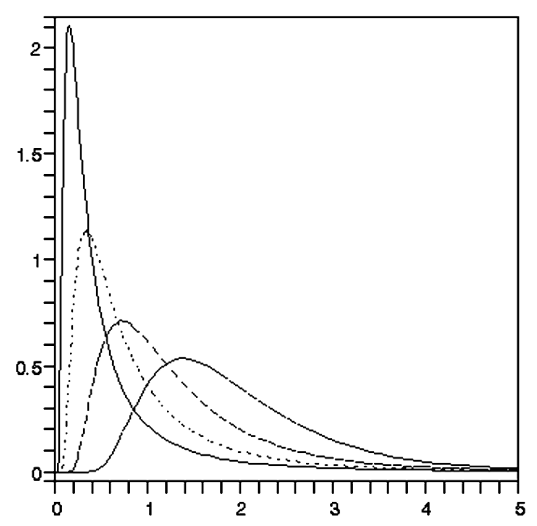

$\lambda=1 / 2$

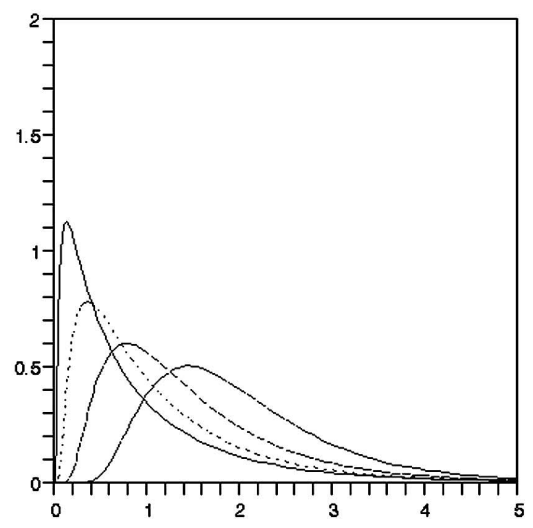

$\lambda=0$

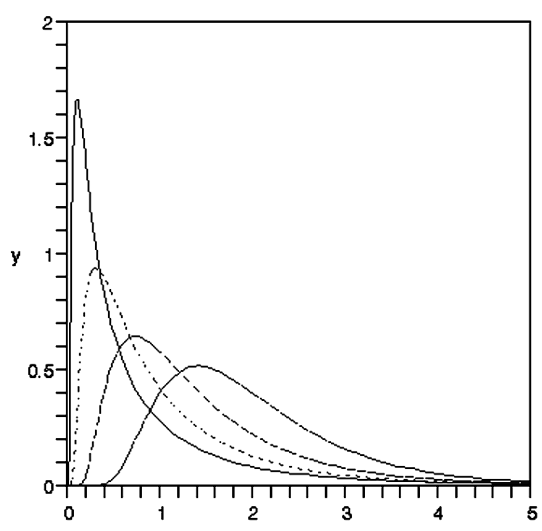

$\lambda=1$

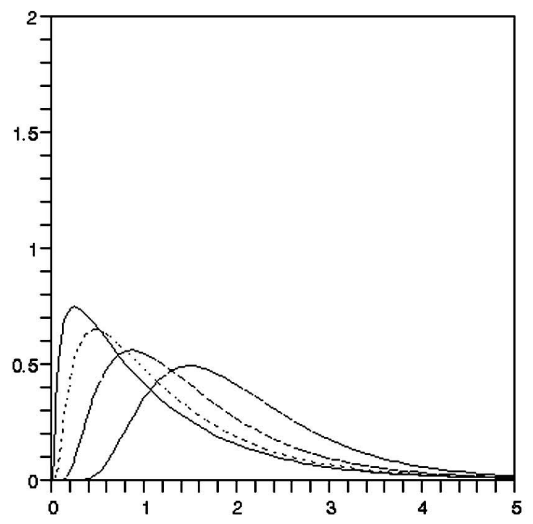

Figure 1: Some GIG densities for different values of $\lambda$ and $\omega=\delta \gamma$.

The mean and the variance of a GIG random variable $X$ can easily be obtained from the Laplace transform. They are given respectively by

$$
\mathbb{E}[X]=\frac{\delta}{\gamma} \frac{K_{\lambda+1}(\delta \gamma)}{K_{\lambda}(\delta \gamma)}
$$

and

$$
\operatorname{Var}[X]=\frac{\delta^{2}}{\gamma^{2}}\left[\frac{K_{\lambda+2}(\delta \gamma)}{K_{\lambda}(\delta \gamma)}-\frac{K_{\lambda+1}^{2}(\delta \gamma)}{K_{\lambda}^{2}(\delta \gamma)}\right]
$$

Barndorff-Nielsen and Halgreen (1977) showed that the GIG distribution is infinitely divisible. We use this property to construct a Lévy process in the following section. 


\section{Generalized Inverse Gaussian Lévy Process}

Let $\left(\Omega, F,(F)_{t \geq 0}, \mathbb{P}\right)$ be a filtered probability space. All the stochastic processes in the paper are assumed to be in this filtered probability space.

Definition 3.1. An adapted càdlàg $\mathbb{R}$-valued process $X=\{X(t)\}_{t \geq 0}$ with $X(0)=0$ is a Lévy process if and only if its characteristic function is of the form $\phi_{t}(s)=$ $e^{-t \varphi(s)}$ where

$$
\varphi(s)=-i a s+\frac{b^{2}}{2} s^{2}+\int_{\mathbb{R}_{0}}\left[1-e^{i s x}+i s x \rrbracket_{\{(-1,1)\}}(x)\right] v(d x), \quad s \in R,
$$

while $a, b \in \mathbb{R}$ and $v$ is a positive measure on $\mathbb{R}_{0}=\mathbb{R}-\{0\}$ satisfying

$$
\int_{\mathbb{R}_{0}} 1 \wedge|x|^{2} v(d x)<\infty
$$

The parameters $a, b^{2}$ and $v$ uniquely determine $X$. The measure $v$ is called the Lévy measure and the exponent $\varphi$ is called the characteristic exponent of the process $X$.

This class of processes is in one-to-one correspondence with the class of infinitely divisible distributions. Every infinitely divisible distribution generates a Lévy process and the increments of every Lévy process are infinitely divisible distributed.

The Lévy measure $v$ governs the occurrence of the jumps of the process $X$. If $b^{2}>0$ and the Lévy measure is identically zero then the process is a Brownian motion (the only continuous Lévy process). If the Gaussian coefficient $b^{2}=0$, the process is entirely composed of jumps, if in addition $\int_{\mathbb{R}_{0}} v(d x)<\infty$ then the process is a compound Poisson process where the distribution of the jumps is $\frac{v(d x)}{\int_{\mathbb{R}} v(d x)}$ and the jumps epochs occur at rate $\int_{\mathbb{R}} v(d x)$. On the other hand, if $\int_{\mathbb{R}_{0}} v(d x)=\infty$ and $\int_{\mathbb{R}_{0}} 1 \wedge|x| v(d x)<\infty$, then the process has an infinite number of small jumps but is of finite variation. Finally, if $\int_{\mathbb{R}_{0}} v(d x)=\infty$ and $\int_{\mathbb{R}_{0}} 1 \wedge|x|$ $v(d x)=\infty$, the process has infinitely many jumps and is of unbounded variation.

If the Gaussian coefficient $b^{2}=0$ and if the Lévy measure $v$ is defined on $(0, \infty)$ such that $\int_{0}^{\infty}(1 \wedge x) v(d x)<\infty$ then the corresponding Lévy process is called a subordinator. Its increments are always positive.

If $b^{2}=0$ and the Lévy measure $v$ satisfies

$$
\int_{\mathbb{R}_{0}}(1 \wedge|x|) v(d x)<\infty
$$

then the process $X$ is of finite variation and we can discard the centering function in (7) and simply write

$$
\varphi(s)=\Psi(-i s)=-i a s+\int_{\mathbb{R}_{0}}\left[1-e^{i s x}\right] v(d x), \quad s \in R .
$$


The alternative function $\Psi$ is called the Laplace exponent of the process. For an account of the theory we refer to Sato (1999) or Bertoin (1996) and for recent applications to Barndorff-Nielsen, Mikosh and Resnick (2001).

Notice that the general aggregate claims process $S$ defined by (3) has a characteristic exponent of the form (9) with $Q(x)=\int_{x}^{\infty} q(s) d s$ where $q(d s)=-d Q(s)$ is the Lévy measure. This is because the measure $q(d s)$ is defined on the positive axis and the increments of the process can only be positive making $S$ a subordinator. Also notice that the jumps of $S$ larger than $\varepsilon$ form a compound Poisson process with jump rate $Q(\varepsilon)$ and jump density $\frac{q(d x)}{Q(\varepsilon)}$.

In the spirit of Dufresne, Gerber and Shiu (1991) we define the generalized inverse Gaussian Lévy process in terms of a non-negative and non-increasing function $Q$, which, as we have seen, is the Lévy measure of the process. In Barndorff-Nielsen and Shephard (2001) or Schoutens (2003) we can find that the Lévy measure in the Lévy-Khintchine representation of the $\operatorname{GIG}(\lambda, \delta, \gamma)$ distribution is

$$
q(d x)=\frac{1}{x}\left[\delta^{2} \int_{0}^{\infty} e^{-x t} g_{\lambda}\left(2 \delta^{2} t\right) d t+\max \{0, \lambda\}\right] e^{-\gamma^{2} x / 2} d x, \quad x>0,
$$

where

$$
g_{\lambda}(y)=\left\{\frac{\pi^{2}}{2} y\left[J_{|\lambda|}^{2}(\sqrt{y})+N_{|\lambda|}^{2}(\sqrt{y})\right]\right\}^{-1}, \quad y>0 .
$$

$J$ and $N$ are modified Bessel functions.

Since the GIG distribution is infinitely divisible and with support on the positive axis we can define a positive Lévy process $S_{G I G}$ described by its characteristic function $\phi_{t}(s)=e^{-t \Psi_{G I G}(-i s)}$ where $\Psi_{G I G}$ is the Laplace exponent of the GIG distribution, i.e.,

$$
\Psi_{G I G}(s)=\int_{\mathbb{R}_{0}}\left[e^{-s x}-1\right] q(d x), \quad s \in \mathbb{R} .
$$

This last equation (11) is of the form (3) with $Q(x)=\int_{x}^{\infty} q(d t)$. Notice that since $S_{G I G}$ is a subordinator, its Lévy measure satisfies $\int_{0}^{\infty} x q(d x)<\infty$. Moreover, $Q(0)=\int_{0}^{\infty} q(d x)=\infty$ and the process $S_{G I G}$ is composed of an infinite number of small jumps.

Such a process is a generalization of the gamma and inverse Gaussian process. From the form of its Laplace transform we can see that GIG distributions are not closed under convolutions. It follows that all increments of length one follow a GIG distribution. However, increments of other lengths follow an infinitely divisible distribution that does not belong to the GIG class. This is because, by construction, $\mathbb{E}\left[e^{i z S_{G I G}(t)}\right]=\phi_{t}(z)=\left[\phi_{1}(z)\right]^{t}$.

Nonetheless, we can compute this class of infinitely divisible densities using the Fourier inversion formula

$$
f_{t}(x)=\frac{1}{\pi} \int_{0}^{\infty} \cos (u x) \phi_{t}(u) d u .
$$




\section{Ruin Theory for the GIG Lévy Process}

A general risk model based on a GIG aggregate claims process would be

$$
U(t)=u+c t-S_{G I G}(t), \quad t \geq 0,
$$

where $S_{G I G}$ is a generalized inverse Gaussian Lévy process, $u$ is the initial surplus, $c$ is a constant premium rate defined as $c=(1+\theta) \mathbb{E}\left[S_{G I G}(1)\right]$ where $\theta$ is the security loading factor. The risk process defined in (13) belongs to the family of processes discussed in Morales and Schoutens (2003). For an account on the classical risk model we refer to Grandell (1991).

Chaubey et al. (1998) show that the inverse Gaussian distribution provides a good fit for aggregate claims for a wide choice of claim size distributions. The extra parameter of the GIG distribution might make it a more flexible distribution to model aggregate claims. This is yet to be explored, however it provides a motivation to model aggregate claims with a GIG distribution.

Despite the fact that such an aggregate claims process has an infinite number of small claims in any interval, it is a of finite variation and the claims remain small enough as to assure that the maximum aggregate loss is still a compound geometric random variable.

For the process (13), the maximum aggregate loss is the random variable defined by

$$
L=\sup _{t>0}\left\{S_{G I G}(t)-c t\right\}
$$

and the ruin probability is

$$
\psi(u)=P\left\{\inf \left[t>0 \mid u+c t-S_{G I G}(t)<0\right]<\infty\right\} \quad u \geq 0 .
$$

In the classical compound Poisson model [see Grandell (1991)], $L$ is related to the ruin probability $\psi$ as indicated by the following relation:

$$
\psi(u)=\mathbb{P}(L>u), \quad u \geq 0,
$$

i.e., the ruin probability $\psi$ is the tail of the distribution of the maximum aggregate loss. Moreover, $L$ is a compound geometric random variable with parameter $\frac{1}{1+\theta}$ and jump distribution given by

$$
F(x)=\frac{\int_{0}^{x}[1-G(y)] d y}{\int_{0}^{\infty}[1-G(y)] d y}, \quad x>0,
$$

where $G$ is the claim distribution of the original compound Poisson risk process. This last fact, along with (16) implies the following equation for the ruin probability in the classical case

$$
\psi(u)=\frac{\theta}{1+\theta} \sum_{n=1}^{\infty}\left(\frac{1}{1+\theta}\right)^{n}\left[1-F^{*(n)}(u)\right], \quad u \geq 0,
$$


where $F^{*(n)}$ indicates the $n$-fold convolution of $F$.

For the GIG process we cannot talk about a claim size distribution equivalent to $G$ in (17). However, all of these relations are preserved if the aggregate claims process is a GIG Lévy process. This result was already pointed out by Dufresne et al. (1991) and a formal proof for a more general model can be found in Yang and Zhang (2001). Here we give another proof within the framework of the theory of Lévy processes.

Theorem 4.1. Let $U$ be the process in (13). Also let $L$ and $\psi$ be as in (14) and (15) respectively. Then, the ruin probability $\psi$ is related to L as in (16). Moreover, $L$ is a compound geometric random variable of the form

$$
L=\sum_{i=1}^{M} L_{i}
$$

where $M$ is a geometric random variable with with probability function

$$
p(k)=\frac{\theta}{1+\theta}\left(\frac{1}{1+\theta}\right)^{k}, \quad k \geq 0,
$$

and $\left\{L_{i}\right\}_{i=1,2, \ldots}$ are i.i.d. random variables with density

$$
m(x)=\frac{Q(x)}{\int_{0}^{\infty} x d Q(x)}, x>0 .
$$

Recall that $Q$ is the Lévy measure of the GIG Lévy process with density given by (10).

Proof. This is a direct consequence of the fact that $S_{G I G}$ is a limit of a sequence of compound Poisson processes as in (4).

The converging sequence $\left\{S_{\varepsilon}\right\}_{\varepsilon}>0$ is defined by its characteristic function

$$
\phi_{t}^{(\varepsilon)}(z)=e^{t \int_{0}^{\infty}\left[e^{i z x}-1\right] d Q_{\varepsilon}(x)}, \quad z>0,
$$

where $q_{\varepsilon}(d x)=-d Q_{\varepsilon}(x)=-\mathbb{\square}_{[\varepsilon, \infty)}(x) d Q(x)$. Therefore, the claim rate is $\int_{0}^{\infty} q_{\varepsilon}(x) d x$ and the claim size density is $\frac{d Q_{\varepsilon}(x)}{\int_{0}^{\infty} d Q_{\varepsilon}(s)}$.

For such a compound Poisson process the maximum aggregate loss $L_{\varepsilon}$ is a compound geometric r.v. of the form

$$
L_{\varepsilon}=\sum_{i=0}^{M} L_{i}^{\varepsilon},
$$

and it has the following characteristic function [see Asmussen (2000)]:

$$
\phi_{\varepsilon}(z)=\frac{\frac{\theta}{1+\theta}}{1-\frac{1}{1+\theta} \phi_{L_{i}^{\varepsilon}}(z)}, \quad z>0,
$$


where $\phi_{L_{i}^{\varepsilon}}$ is the characteristic function of the random variable $L_{i}^{\varepsilon}$ with density

$$
m_{\varepsilon}(x)=\frac{Q_{\varepsilon}(x)}{\int_{0}^{\infty} s q_{\varepsilon}(s) d s}, \quad x>0 .
$$

This is the classical ladder-height decomposition for the classical risk process. Moreover, we have that the sequence of ruin probabilities $\psi_{\varepsilon}$ is such that

$$
\psi_{\varepsilon}(u)=\mathbb{P}\left(L_{\varepsilon}>u\right), \quad u>0 .
$$

Now, notice that the density (19) converges to

$$
m(x)=\frac{Q(x)}{\int_{0}^{\infty} s q(s) d s}, \quad x>0,
$$

as $\varepsilon \longrightarrow 0$. This is because $S_{G I G}$ is a subordinator and $\int_{0}^{\infty} x q(x) d x<\infty$. In consequence, its characteristic function $\phi_{L_{i}^{\varepsilon}}$ converges to the characteristic function $\phi_{L_{i}}$ of the density $m$. In light of this we have that the characteristic function (18) converges to

$$
\phi(z)=\frac{\frac{\theta}{1+\theta}}{1-\frac{1}{1+\theta} \phi_{L_{i}}(z)}, \quad z>0,
$$

as $\varepsilon \longrightarrow 0$. This last equation is the characteristic function of a compound geometric random variable with rate $\frac{\theta}{1+\theta}$ and with jump distribution given by (21). Further, because of equation (20), we have that the ruin probability $\psi$ is the tail of the compound geometric distribution described by (22). This completes the proof.

Remark 4.1. The result in Theorem 4.1 is valid for a wider class of Lévy processes as pointed out by Dufresne et al. (1991). In fact, it is true for any risk model with a subordinator as the aggregate claims process.

In practice, the fact that the ladder height distribution (21) is given in terms of the integral (10) might be seen as a setback. However this integral might be computed numerically or as Dufresne, Gerber and Shiu (1991) had already pointed out for the gamma process, this property leads to upper and lower bounds for the ruin probability. We can also find in Morales and Schoutens (2003) extensions of the notion of Lundberg bounds to a more general risk model based on Lévy processes. These two approaches are further discussed for a GIG risk model in the following sections. 


\section{LUNDBERG-LiKe UPPER BOUNDS FOR THE GIG RISK PROCESS}

It is known that for (17) we can construct an exponential upper bound for the associated ultimate ruin probability of the form [see Feller (1971) or Asmussen (2000)]:

$$
\psi(u) \leq e^{-R u}, \quad u \geq 0,
$$

where $R$ is the positive solution of $\Psi_{U-u}(r)=0$. Recall that the function $\Psi_{U-u}$ is the Laplace exponent of the process $U(t)-u=c t-S(t)$ defined in (13).

In this case we have that this generalized Lundberg equation takes the form:

$$
c r=\ln \left[\mathbb{L}_{G I G}(-r)\right],
$$

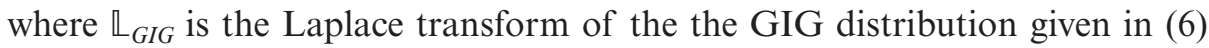
and $c$ is the loaded premium defined as $c=(1+\theta) \mathbb{E}\left[S_{G I G}\right]$. This comes from the fact that $\Psi_{S}(r)=\ln \mathbb{L}_{G I G}(r)$.

We can verify that (24) has a non-trivial positive solution. Recall that

$$
\left.\Psi_{S}^{\prime}(-r)\right|_{r=0}=\left.\frac{d}{d r} \ln \mathbb{L}_{G I G}(-r)\right|_{r=0}=\mathbb{E}\left[S_{G I G}\right] .
$$

Then, since $\Psi(-r)$ is a convex function, equation (24) has a positive solution if $c>\left.\Psi_{S}^{\prime}(-r)\right|_{r=0}=\mathbb{E}\left[S_{G I G}\right]$. Now, recall that, in order to meet the net profit condition, we have defined

$$
c=(1+\theta) \mathbb{E}\left[S_{G I G}\right]>\mathbb{E}\left[S_{G I G}\right] .
$$

This implies that (24) has a non-trivial positive solution.

Unfortunately, there is no closed form expression for the solution $R$ of (24). The generalized Lundberg equation (24) can be explicitely written as

$$
e^{c r}=\frac{K_{\lambda}\left(\delta \gamma \sqrt{1-\frac{2 r}{\gamma^{2}}}\right)}{K_{\lambda}(\delta \gamma)\left(1-\frac{2 r}{\gamma^{2}}\right)^{-\lambda / 2}},
$$

for $r<\gamma^{2} / 2$ if $\lambda \geq 0$ or $r \leq \gamma^{2} / 2$ if $\lambda<0$. We can see that the modified Bessel function appearing in the equation makes it impossible to obtain a closed form for $R$. However we can numerically compute $R$ using available mathematical software such as Maple. We will illustrate this in a final section regarding numerical results.

In the following section we illustrate some particular cases of the GIG risk process for which further analysis can be carried on.

\section{Ruin Probabilities for the GIG Risk Process}

Theorem 4.1 shows that the ladder height decomposition is still valid for the GIG risk process. This allows us to derive equations analogous to those in the classical case. For instance, the ruin probability (15) satisfies 


$$
\psi(u)=\frac{\theta}{1+\theta} \sum_{n=1}^{\infty}\left(\frac{1}{1+\theta}\right)^{n}\left[1-M^{*(n)}(x)\right], \quad u \geq 0,
$$

where $M$ is the distribution function of the density $m$ in (21). This last equation is the analogous to equation (17).

Furthermore, equation (25) implies that $\psi$ satisfies the following renewal equation

$$
\psi(u)=\frac{1-M(u)}{1+\theta}+\frac{1}{1+\theta} \int_{0}^{u} \psi(u-y) d M(y), \quad u \geq 0 .
$$

Just as in the classical case, the computation of (25) and (26) is complicated. This situation is aggravated by the form of the distribution $M$. Recall that

$$
m(x)=\frac{Q(x)}{\int_{0}^{\infty} t[-d Q(t)]}=\frac{\int_{x}^{\infty} q(d t)}{\int_{0}^{\infty} t q(t) d t}, \quad x \geq 0,
$$

where $q(d x)$ is the Lévy measure of the GIG process given by (10) and that involves all three modified Bessel functions.

However, we can compute bounds for the ruin probability (15). In Dufresne et al. (1991), they compute bounds for the gamma process using a discretization method suggested in Dufresne and Gerber (1989). This recursive method can be implemented for any values of $u$ in a GIG risk model despite the numerical integrations involved. We compute these bounds for a numerical example in Section 7.

We can also compute analytical bounds using existing results for compound geometric tails. For instance, Cai and Garrido (1998) give lower and upper bounds for ruin probabilities satisfying equations of the form (25). These bounds, for $u \geq 0$, are given by

$$
\frac{\bar{M}(u)}{\theta+\bar{M}(u)} \leq \psi(u) \leq \frac{\bar{M}(u)+\mathbb{E}(L) M(u) / u}{1+\theta+\mathbb{E}(L) M(u) / u},
$$

where $\bar{M}(x)=1-M(x)$ and $\mathbb{E}(L)$ is the expected value of the maximum aggregate loss random variable. We have gone around the problem of computing convolutions of $M$ by using analytical bounds, this will have an impact in the accuracy of these bounds.

Regardless the type of bounds we want to implement, we still have to deal with $\bar{M}(x)$ and $\mathbb{E}(L)$. These two functions are given in terms of the Lévy measure $(10)$ as follows:

$$
\bar{M}(x)=\frac{\int_{x}^{\infty} \int_{y}^{\infty} \frac{1}{\xi}\left[\delta^{2} \int_{0}^{\infty} e^{-\xi t} g_{\lambda}\left(2 \delta^{2} t\right) d t+\max \{0, \lambda\}\right] e^{-\gamma^{2} \xi / 2} d \xi d y}{\int_{0}^{\infty}\left[\delta^{2} \int_{0}^{\infty} e^{-x t} g_{\lambda}\left(2 \delta^{2} t\right) d t+\max \{0, \lambda\}\right] e^{-\gamma^{2} x / 2} d x}, x \geq 0,
$$


and

$$
\mathbb{E}(L)=\frac{1}{\theta} \int_{0}^{\infty} \bar{M}(x) d x,
$$

where

$$
g_{\lambda}(y)=\left\{\frac{\pi^{2}}{2} y\left[J_{|\lambda|}^{2}(\sqrt{y})+N_{|\lambda|}^{2}(\sqrt{y})\right]\right\}^{-1}, \quad y>0 .
$$

Integrals involving modified Bessel functions can be computed numerically. Here, we illustrate the particular cases $\lambda=1 / 2$ and $\lambda=-1 / 2$. For these choices of the parameter $\lambda$, the function $g_{\lambda}$ takes a much simpler form thanks to the following relations [see Abramowitz and Stegun (1970)]

$$
J_{\frac{1}{2}}(y)=\sqrt{\frac{2}{\pi y}} \sin (y) \text { and } N_{\frac{1}{2}}(y)=-\sqrt{\frac{2}{\pi y}} \cos (y) .
$$

This yields

$$
g_{\frac{1}{2}}(y)=g_{-\frac{1}{2}}(y)=\left\{\frac{\pi^{2}}{2} y\left[J_{\frac{1}{2}}^{2}(\sqrt{y})+N_{\frac{1}{2}}^{2}(\sqrt{y})\right]\right\}^{-1}=\frac{1}{\pi \sqrt{y}} .
$$

Using (30) we get that, for $\lambda=1 / 2$, equation (28) becomes

$$
\begin{aligned}
\bar{M}(x) & =\frac{\int_{x}^{\infty} \int_{y}^{\infty} \frac{1}{\xi}\left[\delta^{2} \int_{0}^{\infty} \frac{e^{-\xi} t}{\pi \delta \sqrt{2 t}} d t+\frac{1}{2}\right] e^{-\gamma^{2} \xi / 2} d \xi d y}{\int_{0}^{\infty}\left[\delta^{2} \int_{0}^{\infty} \frac{e^{-x t}}{\pi \delta \sqrt{2 t}} d t+\frac{1}{2}\right] e^{-\gamma^{2} x / 2} d x} \\
& =\frac{\int_{x}^{\infty} \int_{y}^{\infty} \frac{1}{\xi}\left[\delta^{2} \frac{\Gamma(1 / 2)}{\pi \delta \sqrt{2 \xi}}+\frac{1}{2}\right] e^{-\gamma^{2} \xi / 2} d \xi d y}{\int_{0}^{\infty}\left[\delta^{2} \frac{\Gamma(1 / 2)}{\pi \delta \sqrt{2 x}}+\frac{1}{2}\right] e^{-\gamma^{2} x / 2} d x} \\
& =\frac{\int_{x}^{\infty}\left[\frac{\delta \Gamma(1 / 2)}{\pi \sqrt{2}} \int_{y}^{\infty} \xi^{-3 / 2} e^{-\gamma^{2} \xi / 2} d \xi+\frac{1}{2} \int_{y}^{\infty} \xi^{-1} e^{-\gamma^{2} \xi / 2} d \xi\right] d y}{\frac{\delta \Gamma(1 / 2)}{\pi \sqrt{2}} \int_{0}^{\infty} x^{-1 / 2} e^{-\gamma^{2} x / 2} d x+\frac{1}{2} \int_{0}^{\infty} e^{-\gamma^{2} x / 2} d x} \\
& =\frac{\gamma^{2}}{\delta \gamma+1} \int_{x}^{\infty}\left[\frac{\delta \Gamma\left(\frac{1}{2}\right)}{\pi \sqrt{2}} \int_{y}^{\infty} \xi^{-\frac{3}{2}} e^{-\frac{\gamma^{2}}{2} \xi} d \xi+\frac{1}{2} \int_{y}^{\infty} \xi^{-1} e^{-\frac{\gamma^{2}}{2} \xi} d \xi\right] d y .
\end{aligned}
$$

This last equation comes from the fact that $\Gamma\left(\frac{1}{2}\right)=\sqrt{\pi}$ which reduces the denominator to the constant $\frac{\delta_{\gamma}+1}{\gamma^{2}}$. As for the numerator, if we integrate by parts, we can rewrite 


$$
\begin{aligned}
\bar{M}(x)= & \frac{\gamma^{2}}{\delta \gamma+1}\left\{\int_{x}^{\infty} \frac{\delta \Gamma\left(\frac{1}{2}\right)}{\pi \sqrt{2}}\left[2 y^{-\frac{1}{2}} e^{-\frac{\gamma^{2}}{2} y}-\gamma^{2} \int_{y}^{\infty} \xi^{-\frac{1}{2}} e^{-\frac{\gamma^{2}}{2} \xi} d \xi\right] d y\right. \\
& \left.+\int_{x}^{\infty} \frac{1}{2} \int_{y}^{\infty} \xi^{-1} e^{-\frac{\gamma^{2}}{2} \xi} d \xi d y\right\} .
\end{aligned}
$$

The function inside the integral is the ladder height density $m$. If we change the order of integration in (31), it simplifies even further yielding

$$
\begin{aligned}
\bar{M}(x)= & \frac{\gamma^{2}}{\delta \gamma+1}\left\{\frac{2 \delta \Gamma\left(\frac{1}{2}\right)}{\pi \gamma} \bar{\Gamma}\left(\frac{\gamma^{2}}{2} x ; \frac{1}{2}\right)\right. \\
& -\frac{\delta \Gamma\left(\frac{1}{2}\right)}{\pi}\left[\frac{2}{\gamma} \bar{\Gamma}\left(\frac{\gamma^{2}}{2} x ; \frac{3}{2}\right)-\gamma x \bar{\Gamma}\left(\frac{\gamma^{2}}{2} x ; \frac{1}{2}\right)\right] \\
& \left.+\frac{1}{2}\left[\frac{2}{\gamma^{2}} e^{-\frac{\gamma^{2}}{2} x}-x \bar{\Gamma}\left(\frac{\gamma^{2}}{2} x ; 0\right)\right]\right\},
\end{aligned}
$$

where $\bar{\Gamma}(u ; \alpha)=\int_{u}^{\infty} x^{\alpha-1} e^{-x} d x$ is the incomplete gamma function. Notice that $\bar{\Gamma}(u ; 0)$ is the exponential integral function $-E_{i}(-u)$ [see Abramowitz and Stegun (1970)].

Consequently, $\mathbb{E}(L)$ is given by

$$
\mathbb{E}(L)=\frac{1}{\theta} \int_{0}^{\infty} \bar{M}(x) d x
$$

If we substitute $\bar{M}$ by (31) and compute the double integrals by changing the order of integration we have that

$$
\mathbb{E}(L)=\frac{1}{\theta} \frac{\gamma^{2}}{\delta \gamma+1}\left\{\frac{\delta}{2 \gamma^{3}}+\frac{1}{\gamma^{4}}\right\} .
$$

In this last equality we use the fact that $\Gamma\left(\frac{3}{2}\right)=\frac{1}{2} \Gamma\left(\frac{1}{2}\right), \Gamma\left(\frac{5}{2}\right)=\frac{3}{4} \Gamma\left(\frac{1}{2}\right)$ and $\Gamma(2)=1$.

Substituting (32) and (33) into the expressions (27) yields lower and upper bounds for the ruin probability of the GIG risk process with $\lambda=\frac{1}{2}$.

Bounds for the ruin probability for the inverse Gaussian case $\left(\lambda=-\frac{1}{2}\right)$ can be derived in the same manner. The first integral in the numerator and denominator of (28) simplifies since $\max \left\{0,-\frac{1}{2}\right\}=0$ implying the following forms for $\bar{M}$ and $\mathbb{E}(L)$ :

$$
\begin{aligned}
\bar{M}(x)= & \frac{\gamma}{\delta}\left\{\frac{2 \delta \Gamma\left(\frac{1}{2}\right)}{\pi \gamma} \bar{\Gamma}\left(\frac{\gamma^{2}}{2} x ; \frac{1}{2}\right)\right. \\
& \left.-\frac{\delta \Gamma\left(\frac{1}{2}\right)}{\pi}\left[\frac{2}{\gamma} \bar{\Gamma}\left(\frac{\gamma^{2}}{2} x ; \frac{3}{2}\right)-\gamma x \bar{\Gamma}\left(\frac{\gamma^{2}}{2} x ; \frac{1}{2}\right)\right]\right\},
\end{aligned}
$$


and

$$
\mathbb{E}(L)=\frac{1}{\theta} \frac{\gamma}{\delta}\left[\frac{\delta}{2 \gamma^{3}}\right] .
$$

Equations (32) and (34) generalize similar expressions for the gamma and the inverse Gaussian processes in Dufresne et al. (1991).

\section{Numerical Results}

We carry out the numerical evaluation of the bounds defined by (23), (27), (32) and (33) for the case $\lambda=\frac{1}{2}$ as an illustration. The incomplete gamma and exponential integral functions were computed using Maple. There exist also well-known analytical expansions [see Abramowitz and Stegun (1970)]. The solutions of equation (23) were computed using Maple.

\begin{tabular}{|c|c|c|c|c|c|c|c|c|c|}
\hline \multirow[b]{3}{*}{$\mathbf{u}$} & \multicolumn{9}{|c|}{$\theta$} \\
\hline & \multicolumn{3}{|c|}{0.1} & \multicolumn{3}{|c|}{0.2} & \multicolumn{3}{|c|}{0.3} \\
\hline & LOWER & UPPER & UPPER L & LOWER & UPPER & UPPER L & LOWER & UPPER & UPPER L \\
\hline 50 & 0.79096 & 0.92704 & \begin{tabular}{|l}
0.920678 \\
\end{tabular} & 0.65421 & 0.85316 & \begin{tabular}{|l}
0.870315 \\
\end{tabular} & 0.55778 & 0.78214 & 0.837349 \\
\hline 100 & 0.69094 & 0.86701 & 0.847649 & 0.52782 & 0.75250 & 0.757448 & 0.42701 & 0.65610 & \\
\hline 150 & 0.58797 & 0.81380 & 0.780412 & 0.41640 & 0.67171 & 0.659218 & 0.32234 & 0.56281 & 0.58711 \\
\hline 200 & 0.48659 & 0.76625 & & 0.32152 & 0.60531 & & & 0.49078 & 0.491616 \\
\hline 250 & & 0.723 & & 0.24 & 0.54995 & & 0.17 & 0.43379 & 1654 \\
\hline 300 & 0.30914 & 0.68515 & 0.609043 & 0.18283 & 0.50328 & 0.434568 & 0.12980 & 0.38784 & 0.344698 \\
\hline 350 & 0.23914 & 0.65043 & 0.560732 & 0.13581 & 0.46357 & 0.378211 & 0.09483 & 0.35021 & 0.288632 \\
\hline 400 & & & & & 0.42946 & & & 0.31897 & 0.241686 \\
\hline 450 & 0.13765 & 0.59035 & 0.475304 & 0.07391 & 0.39992 & 0.286475 & 0.05052 & 0.29271 & 0.202375 \\
\hline 500 & 0.10315 & 0.56424 & 0.437602 & 0.05438 & 0.37414 & 0.249323 & 0.03692 & 0.27038 & 0.169459 \\
\hline 550 & & & 0.402891 & 0.040 & 0.35146 & & & 0.25120 & 0.141896 \\
\hline 750 & & & & 0.01186 & 0.28299 & & & 0.19579 & 0.069758 \\
\hline 950 & 0.00720 & 0.40379 & 0.207994 & 0.00361 & 0.23705 & 0.071425 & 0.00241 & 0.16064 & 0.034294 \\
\hline
\end{tabular}

TABLE 1: Upper and lower bounds for a GIG risk process for different values of $\theta$. The parameters of the underlying GIG distribution are $\lambda=\frac{1}{2}, \gamma=\frac{1}{10}$ and $\delta=10 \sqrt{2}$.

In Table 1 we present bounds for the ruin probability of a GIG risk process with parameters $\lambda=\frac{1}{2}, \gamma=\frac{1}{2}$ and $\delta=10 \sqrt{2}$ which correspond to an expected aggregate claim value of 142.42 per unit time. Different values for the safety loading $\theta$ are shown. The solutions of equation (23) are $0.00165289,0.00277801$, and 0.00355029 for values of $\theta$ of $0.1,0.2$ and 0.3 respectively. The Lundberg upper bounds are shown under column UPPER L.

We can see that bounds computed using (27) are not bad for small values of $u$. However, we can see that for small values these bounds are still not tight enough. The Lundberg upper bounds are tighter for large values of $u$. This is the region we are particular interested in when we compute ruin probabilities. The discretization method described in Gerber and Dufresne (1989) provides better bounds. 


\begin{tabular}{|c|c|c|c|c|c|c|c|c|c|c|c|c|}
\hline & \multicolumn{4}{|c|}{$\theta=0.1$} & \multicolumn{4}{|c|}{$\theta=0.2$} & \multicolumn{4}{|c|}{$\theta=0.3$} \\
\hline & \multicolumn{2}{|c|}{$\Delta=0.5$} & \multicolumn{2}{|c|}{$A=0.25$} & \multicolumn{2}{|c|}{$A=0.5$} & \multicolumn{2}{|c|}{$\Delta=0.25$} & \multicolumn{2}{|c|}{$A=0.5$} & \multicolumn{2}{|c|}{$A=0.25$} \\
\hline u & lower & upper & lower & upper & lower & upper & lower & upper & lower & upper & lower & upper \\
\hline 1 & 0.90012 & & & & 0.81837 & & & & & & 0.750316 & 0.750634 \\
\hline 10 & 0.875 & & & & & & & & & & & \\
\hline 20 & 0.85812 & & & & & & & & & & & 016 \\
\hline 30 & 0.84317 & 0.84387 & 0.84333 & 0.84368 & 0.727 & & 0.72 & 0.7279 & 0.638 & & 0.638517 & 0.639049 \\
\hline 40 & 0.82963 & 0.8304 & 0.8298 & 0.83019 & 0.70642 & & 0.70666 & 0.70719 & 0.6136 & & 0.61392 & 0.614485 \\
\hline 50 & 0.81701 & & & & & & & & & & & 2212 \\
\hline 60 & 0.80506 & 0.80596 & 0.80527 & 0.80572 & 0.66955 & 67073 & 0.66982 & 0.67042 & 0.570 & 0.571976 & 0.571032 & 0.571647 \\
\hline 70 & 0.79363 & 0.79458 & 0.79385 & 0.79433 & 0.65274 & 0.65398 & 0.65303 & 0.65365 & 0.55 & 0.552779 & 0.551806 & 0.552441 \\
\hline 80 & & & 0.78285 & & & & & & & & & 0.534361 \\
\hline 90 & 0.77196 & 0.77301 & 0.77221 & 0.77273 & 0.62149 & 0.62281 & 0.6218 & 0.62246 & 0.516267 & 0.517597 & 0.516579 & 0.517244 \\
\hline
\end{tabular}

TABLE 2: Upper and lower bounds for a GIG risk process for different values of $\theta$ using an interval of discretization $\Delta$ of 0.5 and 0.25 .

The parameters of the underlying GIG distribution are $\lambda=\frac{1}{2}, \gamma=\frac{1}{10}$ and $\delta=10 \sqrt{2}$.

We computed bounds for the ruin probability using a discretization method (length of interval is 0.5 and 0.25) just like in Dufresne et al. (1991). In Table 2 we can see how we can achieve a higher level of accuracy by refining the interval of discretization. For large values of $u$ the computing time increases because of the recursive nature of the method. This is not a major problem since mathematical software, like Maple, can evaluate the integral functions in (32) and (34) in a reasonable amount of time. Table 2 presents these bounds for small values of $u$.

\section{Conclusions}

We present another example of the risk processes of Dufresne et al. (1991). We illustrate their approach with a wider class of Lévy processes generated by the generalized inverse Gaussian distribution. This family of processes is a generalization of the gamma and inverse Gaussian process first discussed in Dufresne et al. (1991). We showed that they share the same counterintuitive property of having infinitely many claims, and, in spite of which, they accept a ladder height-like decomposition.

The fact that increments over periods of length one follow a GIG distribution makes it interesting for applications since aggregate claims are better fitted by an inverse Gaussian distribution. The extra parameter of the GIG would make a more flexible model for aggregate claims.

This larger class of processes contains the particular cases of the gamma and inverse Gaussian processes explored in Dufresne et al. (1991).

We have shown how Lundberg-like upper bounds can easily be computed. These bounds were seen to be too large (for small values of $u$ ) to be of any practical use. We, then, turned our efforts into using existing bounds in the literature for compound geometric sums. The ladder-height decomposition has been used in the literature [Cai and Garrido (1998)] to produce bounds for the corresponding ruin probability. We implemented these bounds and showed that they are given in terms of Bessel functions. These take on a simple form in the case $\lambda=\frac{1}{2}$. Numerical results for this case are provided. In Table 1 we 
can see that for large values of $u$ these upper bounds are not as sharp as the Lundberg-like bounds.

A discretization method can also be implemented for a GIG risk process. This is illustrated in Table 2 for some values of $u$ and for different values of $\theta$. Despite the integral functions involved in the GIG risk process, this method can still be easily implemented using readily available mathematical software which performs numerical integration of functions like the incomplete gamma or the exponential integral. This method provides bounds for any values of $u$ with a high level of accuracy.

In conclusion, the GIG risk process belongs to the family originally study by Dufresne et al. (1991) and it contains as particular cases the gamma and the inverse Gaussian risk processes. We have seen how despite the counterintuitive property of having an infinite number of small jumps, this process retains basic properties of classical risk processes. This makes it appealing as a risk model. The suitability of GIG distributions as models for aggregate claims is a topic of further research.

\section{ACKNOWLEDGMENT}

I would like to thank Prof. José Garrido at Concordia University and Prof. Elias S.W. Shiu at the University of Iowa for his comments and suggestions on an earlier version of this paper. I acknowledge the financial support of the Society of Actuaries through the Ph.D. Research Grant.

\section{REFERENCES}

Abramowitz, M. and Stegun, I. (1970) Handbook of Mathematical Functions With Formulas, Graphs and Mathematical Tables.

Asmussen, S. (2000) Ruin Probabilities. Advanced Series on Statistical Science and Applied Probability. World Scientific.

BarndorfF-Nielsen, O.E. and Halgreen, C. (1977) Infinite Divisibility of the Hyperbolic and Generalized Inverse Gaussian Distributions. Zeitschrift für Wahrscheinlichkeitstheorie und verwandte Gebiete 38, 439-455.

Barndorff-Nielsen, O.E., Mikosh, T. and Resnick, S., editors (2001) Lévy Processes-Theory and Applications. Birkäuser.

Barndorff-Nielsen, O.E. and Shephard, N. (2001) Non Gaussian OU Based Models and Some of their Uses in Financial Economics. Journal of the Royal Statistical Society. B. 63.

Bertoin, J. (1996) Lévy Processes. Cambridge Tracts in Mathematics. 121. Cambridge University Press.

CAI, J. and Garrido, J. (1998) Aging Properties and Bounds for Ruin Probabilities and Stoploss Premiums. Insurance: Mathematics and Economics 23, 33-43.

Chaubey, Y., Garrido, J. and Trudeau, S. (1998) On the Computation of Aggregate Claims Distributions: Some New Approximations. Insurance: Mathematics and Economics 23, 215230.

Dufresne, F. and Gerber, H.U. (1989) Three Methods to Calculate the Probability of Ruin. ASTIN Bulletin 19, 71-90.

Dufresne, F., Gerber, H.U. and Shiu, E.S.W. (1991) Risk Theory with the Gamma Process. ASTIN Bulletin 21(2), 177-192.

Feller, W. (1971) An Introduction to Probability Theory and Its Applications II. Wiley, New York. 
Grandell, J. (1991) Aspects of Risk Theory. Springer Series in Statistics. Springer-Verlag. JACOD, J. and ShIRYAev, A. (1987) Limit Theorems for Stochastic Processes. Springer-Verlag. Jørgensen, B. (1982) Statistical Properties of the Generalized Inverse Gaussian Distribution. Lecture Notes in Statistics. (9). Springer.

Morales, M. (2003) Generalized Risk Processes and Lévy Modeling in Risk Theory. A Ph.D. thesis in the Department of Mathematics and Statistics. Concordia University. Montréal, Canada.

Morales, M. and Schoutens, W. (2003) A Risk Model Driven by Lévy Processes. Applied Stochastic Models in Business and Industry 19, 147-167.

Sato, K.I. (1999) Lévy Processes and Infinitely Divisible Distributions. Cambridge University Press.

Schoutens, W. (2003) Lévy Processes in Finance: Pricing Financial Derivatives, Wiley.

YANG, H. and Zhang, L. (2001) Spectrally Negative Lévy Processes with Applications in Risk Theory. Advances in Applied Probability 33(1), 281-291.

MANuel Morales

Department of Mathematics and Statistics

York University

N520 Ross Building

4700 Keele St.

Toronto, Ontario

M3J $1 P 3$ - CANADA

E-mail:mmorales@yorku.ca

Phone: (416) 7362100 ext 33768 